\title{
Two genetic disorders (TRMU and SCYL1) explaining transient infantile liver failure in one patient
}

\author{
Teresa Campos $^{1 *}$, Elisa Leão Teles ${ }^{1 *}$, Esmeralda Rodrigues ${ }^{1}$, Célia Nogueira ${ }^{2}$, Laura Vilarinho ${ }^{2}$ and Miguel Leão $^{3}$ \\ ${ }^{1}$ Reference Center of Inherited Metabolic Diseases, Centro Hospitalar Universitário São João, Porto, Portugal \\ ${ }^{2}$ National Institute of Health - Doutor Ricardo Jorge, Porto, Portugal \\ ${ }^{3}$ Department of Genetics, Serviço de Genética, Centro Hospitalar Universitário São João, Porto, Portugal
}

\begin{abstract}
Introduction: Paediatric acute liver failure (PALF) has an extremely heterogeneous aetiology, with some genetic disorders being associated with recurrent/transient episodes of infantile hepatopathy. Here, we report a patient who experienced a severe episode of liver failure with complete recovery, and in whom were discovered two genetic disorders possibly explaining this occurrence: a mitochondrial disease caused by pathogenic variants in TRMU gene and the CALFAN syndrome secondary to mutations in SCYL1 gene.

Case presentation: Healthy female child who was admitted at hospital by the age of 13 months due to acute hepatic failure in context of febrile flu. Hepatotropic infectious diseases were excluded and metabolic evaluation was normal, with exception of positive allopurinol testing. Liver biopsies revealed focal ballooning of hepatocytes and pronounced fibrosis. The liver function gradually recovered. From age of 3 years, she developed intention tremor, and after the age of 10 progressive ataxia and motor-sensory neuropathy. She is now 25 years-old and presents a cerebellar syndrome, without cognitive impairment. There were no further episodes of hepatic failure and serial evaluation showed normal liver function, without evidence of important fibrosis in transient elastography. Genetic analysis revealed that the patient has two novel variants in heterozygosity in TRMU gene, and, in homozygosity, an already known pathogenic variant in SCYL1 gene.
\end{abstract}

Conclusion: Although patient presents neurological features of CALFAN syndrome, it is discussed which genetic disorder (SCYL1 or TRMU) was responsible for the acute liver failure episode.

\section{Introduction}

Paediatric acute liver failure (PALF) is a potentially devastating condition which occurs in previously healthy children of all ages and frequently leads to a rapid clinical deterioration [1]. PALF has heterogeneous aetiology, including drugs/toxics (mainly acetaminophen), infections, auto-immunity, genetic and metabolic disorders. Metabolic disorders account for $10 \%$ of the cases, and the most common causes between these are mitochondrial disorders, urea cycle diseases, fatty acid oxidation defects, classical galactosemia, tyrosinemia type 1 , Niemann-Pick type $\mathrm{C}$ disease and Wilson disease $[2,3]$. For many of these metabolic diseases there is available specific treatment that started precociously could frankly improve the prognosis. In approximately $40 \%$ to $50 \%$ of cases, the cause of PALF remains indeterminate [4]

Recurrent reversible episodes of severe liver injury (RALF), with recovery of hepatic function between crises, are frequently related with heritable disorders, despite being often triggered by virallike illness. $(1,5)$ These inherited aetiologies include mitochondrial diseases, genetic disorders involved in transport of vesicles in the endoplasmic reticulum and Golgi apparatus (NBAS, RINT1, SCYL1), Wolcott-Rallison syndrome, pathogenic variations in dihydrolipoamide dehydrogenase (DLD) and aminoacyl-tRNA synthetases (LARS, MARS) genes [5].

The TRMU gene encodes for the tRNA 5-methylaminomethyl2-thiouridylate methyl-transferase, which is responsible for the 2-thiouridylation (addition of a sulfur-containing thiol group) of mt-tRNA ${ }^{\text {Glu }}$, mt-tRNA ${ }^{\mathrm{Gln}}$ and mt-tRNA ${ }^{\mathrm{Lys}}[6,7]$. Autosomal recessive mutations in the TRMU gene cause abnormal mitochondrial protein translation. While most mitochondrial diseases are progressive conditions, the TRMU deficiency causes reversible infantile liver failure, wherein individuals present with early onset hepatopathy and show complete spontaneous recovery by 2 or 3 years of age, if they survive $[6,8]$.

Recently, mutations in SCYL1 were associated with CALFAN Syndrome, characterized by peripheral neuropathy, cerebellar atrophy, ataxia and recurrent episodes of low $\gamma$-glutamyl-transferase cholestasis and acute liver failure [9]. SCYL1 plays a role in retrograde transport from Golgi to endoplasmic reticulum, as it represents an important protein at the interface between the Golgi apparatus and the membrane trafficking machinery mediated by coatomer (COPI)-coated vesicles [10]. SCYL1 also seems to be involved in the export pathway of nuclear aminoacylation-dependent tRNA export machinery [11]. Here, we report a patient who experienced a severe episode of liver failure with complete spontaneous recovery, and in whom were discovered two genetic disorders possibly explaining this occurrence: the mitochondrial disease due to mutations in TRMU gene and the CALFAN syndrome secondary to mutations in SCYL1 gene.

${ }^{\star}$ Correspondence to: Teresa Isabel Resende de Almeida Campos, Reference Center of Inherited Metabolic Diseases, Centro Hospitalar Universitário São João, 4200-319 Porto, Portugal, Tel: +351962466808; Fax: +351 225025766; E-mail: teresaalmeidacampos@gmail.com

Received: April 10, 2020; Accepted: April 23, 2020; Published: April 30, 2020 


\section{Case report}

A 13-month-old girl, second child of non-consanguineous parents, with an unremarkable personal and familiar past, was admitted in an Intensive Care Unit due to clinical deterioration in context of acute liver failure.

A week before the admission, she presented with flu symptoms (fever and running nose) and started treatment with acetaminophen and acetylsalicylic acid. By the fifth day of fever, it was diagnosed an otitis and amoxicillin and acetominophen was prescribed. But, in the next day, she was revaluated in the hospital due to progressive prostration.

In admission, she was icteric and lethargic and presented an enlarged liver and bruises in the body. Analytical exams confirmed clinical suspicion of acute liver failure, presenting elevation of transaminases, cholestasis, severe impairment of coagulation and hypoglycaemia, without hyperammonaemia. After improvement of general condition and negative viral serologies, she was discharged with suspicion of toxic hepatitis.

During next month post-discharge, parents noticed severe asthenia, some regression in psychomotor development, maintenance of jaundice and reappearance of bruises in members. She was then sent to a Pediatric Unit of Inherited Metabolic Diseases for aetiologic investigation.

Physical exam showed a conscious but quiet child, with icteric skin and sclera, bruises in upper and lower members, hepatomegaly ( $5 \mathrm{~cm}$ below the costal margin), without splenomegaly. Blood tests confirmed liver failure, with conjugated hyperbilirubinemia, elevation of alkaline phosphatase, liver cytolysis, severe alteration of coagulation not corrected by vitamin $\mathrm{K}$. Other diagnostic exams, namely alfa1antitrypsin, copper, ceruloplasmin, alpha fetoprotein, autoantibodies, study of fatty acid oxidation, were normal. It was found orotic aciduria but plasma amino acid profile only showed decrease of aspartic acid and hydroxyproline (these alterations in amino acid profile persisted over the years). In liver biopsy there was diffuse steatosis, ballooning of the cytoplasm of hepatocytes and fibrosis of Disse and portal spaces; analysis of liver mitochondrial respiratory chain complexes was normal. In otherwise, muscle histology was normal but there was partial deficiency (37\%) in complex IV of mitochondrial respiratory chain, that was not confirmed in another biopsy performed 13 years later.

Over the next months she improved, although needing partial restriction of protein intake. There was normalization of liver function, without cholestasis and cytolysis, and no more events of liver failure occurred.

When she was 28 months of age, parents noticed matutinal transient tremor and episodes of sudden apathy, not related with epilepsy. Then, tremor became more intense, interfering with activities of daily living, but without evidence of intelligence impairment. By 6 years of age, she complained of recurrent abdominal pain, but no objective cause was found, and at 9 years, she also referred intermittent pain in members (more in legs), without disturbances at nerve conduction and electromyogram tests. However, over the next 3-4 years, there was evolution for peripheral polyneuropathy and cerebellar syndrome (gait ataxia, severe intention tremor, dysarthria, dysmetria, dysdiadochokinesia). Cerebral MRI showed only slight atrophy of vermis and nerve biopsy evidence of moderate chronic axonal neuropathy. It was also performed extensive panel of metabolic and immunological exams without diagnosis clarification.

At the age of 23 years, molecular studies by Next Generation Sequencing panels were performed. In one panel with 167 nuclear genes involved in mitochondria metabolism, it was identified two novel variants in heterozygosity in gene TRMU: c.10T>G (p.L4V) and c.552C $>\mathrm{T}(\mathrm{p} . \mathrm{A} 184 \mathrm{~A})$, first of uncertain significance and the other considered probable pathogenic by bioinformatic analysis. In otherwise, in a genetic panel including genes related with ataxia, it was recognized premature termination mutation in homozygosity: c.1636C > T (p.Gln546*) in exon 12 of SCYL1 gene.

Now, she is 25 years-old and presents the previous neurologic manifestations, with worsening of tremor, but without cognitive impairment (she is completing her university education). Liver function remains normal and there is no evidence of important fibrosis in transient elastography. The patient maintains evaluation in our reference centre of inherited metabolic diseases, having the transition of care to adult team.

\section{Discussion}

Our patient experienced a transient acute liver failure episode in infancy, with progressive resolution over the following months, what could be explained by the two genetic disorders identified many years later: TRMU and SCYL1. Yet the progressive neurologic manifestations are secondary to the last genetic cause, the acute hepatic insufficiency in infancy is described in both entities.

SCYL1 is a cytoplasmic protein that plays a role in COP1-mediated retrograde protein trafficking and also in nuclear tRNA export [11]. The variant found in SCYL1 gene in homozygosity corresponds to a premature termination mutation (c.1636C $>\mathrm{T}[\mathrm{p}$. Gln546*]) in exon 12 , already described in a female patient, in compound heterozygosity, with a similar clinical presentation [10]. Lenz et al described a pool of seven patients from five families, all with more than one episode of hepatopathy, triggered by febrile infections, and mainly presenting with low $\gamma$-glutamyl-transferase (GGT) cholestatic liver dysfunction, while neurological signs and symptoms varied and had a later onset [9]. Regarding liver histology, there is not a description of a specific pattern in the literature, although most of the cases present portal or bridging fibrosis $[9,10]$. In our patient, we only identified a single occurrence of hepatopathy, as we understand as a continuum between the first two hospital admissions and the total recovery. We do not have information about the level of GGT during crisis, but liver biopsy showed fibrotic tissue remodelling, without progression since then.

The animal model with biallelic disruption of $S c y l 1$ shows neurologic disabilities but does not have the liver dysfunction reported in human patients $[12,13]$. Nevertheless, the conjunction of the different clinical cases allows to consider recurrent liver damage as an important feature of this disease. The pathophysiological mechanism remains unclear but, resembling what possible occurs in mutations in NBAS and RINT1, responsible for early-onset recurrent acute liver failure, it is speculated that the acute hepatic damage could be secondary to dysfunction of the Golgi-ER transport $[5,9,10]$. Otherwise, SCYL1 is also a cytoplasmic component of the mammalian nuclear tRNA export process and so, could share the same mechanism of the cytosolic aminoacyl-tRNA synthetase deficiencies (LARS and MARS), which are associated with multisystem manifestations involving transient infantile hepatopathy [9-11]. 
The TRMU gene encodes for an enzyme responsible for the addition of a sulfur-containing thiol group to some mitochondrial tRNA, namely mt-tRNA ${ }^{\text {Glu }}, m^{-}-\mathrm{tRNA}^{\mathrm{Gln}}$ and mt-tRNA ${ }^{\text {Lys }}$ [6]. Patients mainly present with early onset hepatopathy, but, if they survive the acute phase, there is complete recovery by 2 or 3 years of age, with posterior good evolution [7]. During crisis, serum lactate level is increased and hypoglycaemia is also frequent. Liver biopsies usually reveal, in acute phase, oncocytic change in the hepatocytes, focal ballooning of their cytoplasm and steatosis, and nodular fibrosis in later evaluations. Biochemical analysis of the respiratory chain enzymes in liver show predominant decrease in complex I and complex IV activities [8]. Regarding the TRMU gene, in our patient were identified two novel variants in heterozygosity, one of uncertain significance and the other probable pathogenic by bioinformatic analysis. These alterations possibly promote impairment of TRMU function and also contributed to the event of hepatopathy. Supporting this speculation, there is the fact that, on contrary of what is described in SCYL1 disorder, our patient did not experience recurrent episodes of liver failure, but only one with total recovery by the age of 2-3 years, although with a long period of recuperation with some slight variations. She also presented with hypoglycaemia during crisis (no information available about lactate) and later investigation showed partial deficiency in complex IV of mitochondrial respiratory chain in muscle. The pathologic mechanism by which TRMU and other reversible infantile mitochondrial diseases cause transient manifestations, it is not understood [8]. It is supposed that as TRMU protein requires sulfur for its activity and this derives from cysteine, an essential amino acid in infancy with transitory low levels. Therefore, the combination of temporally low cysteine and the presence of TRMU pathogenic variants in infants leads to low thiouridylation of mt-tRNAs, thereby interfering with mitochondrial protein translation and contributing to disease manifestations [6]. Some papers proposed that early treatment with $\mathrm{L}$-cysteine and $\mathrm{N}$-acetylcysteine may slow disease progression, hasten recovery, and reduce the odds of liver transplantation [6,7].

The diverse analysis of plasma amino acid profile performed to our patient showed decrease of aspartic acid and hydroxyproline. There is not a clear explanation for that, even because hydroxyproline is considered a biomarker of liver disease, with its content positively correlated with the severity of liver fibrosis [14]. We have to wait if the same pattern is found in other patients with SCLY1 or TRMU disorders.

An acute liver failure event is always a challenge, moreover in child, because there is a high probability of being related with a genetic disorder. First, it is imperative to exclude diseases that need specific treatment and, in this group, we include many metabolic disorders that could be easily diagnosed by basic metabolic exams. On the other hand, the number of diseases associated with the occurrence of transient acute liver failure in childhood is increasing, and their identification can help guide patient treatment and follow-up. Thus, some centres, in an early phase of aetiological investigation, also provide genetic panels, besides conventional biochemical and metabolic exams.

We described one of the oldest patients with SCLY1 and TRMU disorders reported in literature. Regardless of the promoter cause of the acute liver failure event, SCYL1 and/or TRMU, hepatic long-term evolution was good, with normal liver function and no progression of fibrosis. Otherwise, concerning neurologic symptoms related with SCYL1 disorder, they seemed to evolve more rapidly during the growth period, with more slow progression since late adolescence.

\section{Acknowledgement}

We acknowledge Dr Isabel Alonso, from UnIGENe and CGPP, IBMC - Instituto de Biologia Molecular e Celular; i3S - Instituto de Investigação e Inovação em Saúde, Universidade do Porto, who performed analysis of SCYL1 gene.

\section{Informed consent}

Written informed consent was obtained from the patient.

\section{Conflict-of-interest statement}

The authors have no conflicts of interest to declare.

\section{Author Contributions}

Teresa Campos and Elisa Leão Teles are first co-authors, they were responsible for the collection of clinical information and wrote the article; Célia Nogueira and Laura Vilarinho managed metabolic and some genetic studies; Esmeralda Rodrigues and Miguel Leão provided relevant clinical information; all the authors revised the text.

\section{References}

1. Alonso E, Horslen S, Behrens E, Doo E (2017) Pediatric acute liver failure of undetermined cause: A research workshop. Hepatology 65: 1026-1037. [Crossref]

2. Squires R, Schneider B, Bucuvales J, Alonso E, Sokol R, et al.(2006) Acute Liver Failure in Children: The First 348 Patients in The Pediatric Acute Liver Failure Study Group. J Pediatr 148: 652-658. [Crossref]

3. Sundaram S, Alonso E, Narkewicz M, Zhang S, Squires R, et al. (2011) Characterization and outcomes of young infants with acute liver failure. J Pediatr 159: 813-818. [Crossref]

4. Narkewicz M, Dell-Olio D, Karpen S, Murray K, Schwarz K, et al. (2009) Pattern of diagnostic evaluation for the causes of pediatric acute liver failure: An opportunity for quality improvement. J Pediatr 155: 801-806. [Crossref]

5. Cousin MA (2019) RINT1 Bi-allelic Variations Cause Infantile-Onset Recurrent Acute Liver Failure and Skeletal Abnormalities. Am J Hum Genet 105: 108-121. [Crossref]

6. Soler-Alfonso C, Pillai N, Cooney E, Mysored K, Boyera S, et al. (2019) L-Cysteine supplementation prevents liver transplantation in a patient with TRMU deficiency. Mol Genet Metab Rep 19:100453

7. Boczonadi V, Smith P, Pyle A, Gomez-Duran A, Schara U, et al. (2013) Altered 2-thiouridylation impairs mitochondrial translation in reversible infantile respiratory chain deficiency. Hum Mol Genet 15: 4602-4615

8. Boczonadi V, Bansagi B, Horvath R (2015) Reversible infantile mitochondrial diseases $J$ Inherit Metab Dis 38: 427-35. [Crossref]

9. Lenz D, McClean P, Kansu A, Bonnen P, Ranucci G, et al. (2018) SCYL1 variants cause a syndrome with low $\gamma$-glutamyl-transferase cholestasis, acute liver failure, and neurodegeneration (CALFAN). Genet Med 20: 1255-1265. [Crossref]

10. Schmidt W, Rutledge S, Schüle R, Mayerhofer B, Züchner S, et al. (2015) Disruptive SCYL1 mutations underlie a syndrome characterized by recurrent episodes of liver failure, peripheral neuropathy, cerebellar atrophy, and ataxia. Am J Hum Genet 97: 855-861. [Crossref]

11. Chafe S, Mangroo D (2010) Scyl1 facilitates nuclear tRNA export in mammalian cells by acting at the nuclear pore complex. Mol Biol Cell 21: 2483-2499.

12. Smith ED (2017) Classification of genes: Standardized clinical validity assessment of gene-disease associations aids diagnostic exome analysis and reclassifications. Hum Mutat 38: 600-608.

13. Schmidt WM (2007) Mutation in the Scyll gene encoding amino-terminal kinase-like protein causes a recessive form of spinocerebellar neurodegeneration. EMBO Rep 8: 691-697. [Crossref]

14. Gabr SA, Alghadir AH, Sherif YE, Ghfar AA (2017) Hydroxyproline as a Biomarker in Liver Disease. In: Patel V, Preedy V [Ed's] Biomarkers in Liver Disease. Biomarkers in Disease: Methods, Discoveries and Applications. Springer, Dordrecht.

Copyright: C2020 Campos T. This is an open-access article distributed under the terms of the Creative Commons Attribution License, which permits unrestricted use, distribution, and reproduction in any medium, provided the original author and source are credited. 